\title{
Effects of Reward and Behavioral Context on Neural Activity in the Primate Inferior Colliculus
}

\author{
Ryan R. Metzger, Nathaniel T. Greene, Kristin Kelly Porter, and Jennifer M. Groh \\ Department of Psychological and Brain Sciences and Center for Cognitive Neuroscience, Dartmouth College, Hanover, New Hampshire 03755
}

\begin{abstract}
Neural activity in the inferior colliculus (IC) likely plays an integral role in the processing of various auditory parameters, such as sound location and frequency. However, little is known about the extent to which IC neural activity may be influenced by the context in which sounds are presented. In this study, we examined neural activity of IC neurons in the rhesus monkey during an auditory task in which a sound served as a localization target for a saccade. Correct performance was rewarded, and the magnitude of the reward was varied in some experiments. Neural activity was also assessed during a task in which the monkey maintained fixation of a light while ignoring the sound, as well as when sounds were presented in the absence of any task. We report that neural activity increased late in the trial in the saccade task in $58 \%$ of neurons and that the level of activity throughout the trials could be modulated by reward magnitude for many neurons. The late-trial neural activity similarly increased in the fixation task in $39 \%$ of the neurons tested for this task but was not observed when sounds were presented in the absence of a behavioral task and reward. Together, these results suggest that a rewardrelated signal influences neural activity in the IC.
\end{abstract}

Key words: monkey; inferior colliculus; context; reward; sound localization; saccade

\section{Introduction}

Perception is a constructive process that depends not only on the physical properties of a stimulus but also on the context in which it occurs: a "context" that includes many factors, such as the immediate relevance of the stimulus to the observer, the presence of related stimuli in other sensory modalities, as well as the observer's current state of arousal, motivation, focus of attention, past experience, and expectation of future events. Previous neurophysiological investigations into this topic have generally focused at the cortical level. However, the existence of ample descending anatomical projections from higher (e.g., cortical) to lower (e.g., subcortical) brain areas suggests that it is possible for lower sensory brain areas to be influenced by factors other than the physical properties of the stimulus and suggests a need for additional scrutiny into the effects of context on physiological processing in "early" sensory areas.

Accordingly, we sought to investigate the effects of context on the processing of sound in the primate inferior colliculus (IC), a structure that is situated reasonably early in the ascending auditory pathway. Early physiological evidence that suggested primate IC neurons might do more than just transmit the physical

Received Dec. 19, 2005; revised May 31, 2006; accepted June 2, 2006.

This work was supported by National Science Foundation Grant 0415634, McKnight Endowment Fund for Neuroscience, Whitehall Foundation, John Merck Scholars Program, and EJLB Foundation (J.M.G.), and National Institutes of Health Grants DC05292 (R.R.M.) and NS44666-03 (K.K.P.). We thank Abigail Underhill and Uri Werner-Reiss for technical assistance and helpful comments.

Correspondence should be addressed to Jennifer M. Groh at her present address: Departments of Neurobiology, and Psychology and Neuroscience, Center for Cognitive Neuroscience, Duke University, Levine Science Research Center, Room B203, Box 90999, Durham, NC 27708. E-mail: jmgroh@duke.edu.

K. K. Porter's present address: University of Alabama at Birmingham, School of Medicine, Birmingham, AL 35294 DOI:10.1523/JNEUROSCI.5401-05.2006

Copyright $\odot 2006$ Society for Neuroscience $\quad$ 0270-6474/06/267468-09\$15.00/0 properties of sound to higher brain structures was reported by Ryan and Miller (1977), who found that discharge rates and latencies of auditory responses were modified by the performance of a task. In addition to receiving ascending auditory signals, the mammalian IC is also the recipient of descending connections from auditory cortex (Diamond et al., 1969; FitzPatrick and Imig, 1978; Adams, 1980; Andersen et al., 1980; Coleman and Clerici, 1987; Winer et al., 2002; Bajo and Moore, 2005) and thalamic nuclei (Adams, 1980; Kuwabara and Zook, 2000; Winer et al., 2002), as well as projections from the retina (Itaya and Van Hoesen, 1982; Yamauchi and Yamadori, 1982; Paloff et al., 1985), visual cortex (Cooper and Young, 1976), and superior colliculus (SC) (Harting, 1977; Adams, 1980; Coleman and Clerici, 1987; Doubell et al., 2000; Winer et al., 2002). Connections to the IC from areas associated with the limbic system such as the central gray (Adams, 1980; Coleman and Clerici, 1987; Larue et al., 2005), amygdala (Marsh et al., 2002), and intralaminar thalamic nuclei (Winer et al., 2002) have also been reported, in addition to modulatory influences by serotonergic inputs (Hurley and Pollak, 1999, 2001, 2005a,b). Together, these studies suggest that auditory signals in this structure are influenced by a variety of nonauditory signals.

To test this hypothesis, we examined the neural activity of IC auditory neurons during an auditory saccade task in which the animal executed a saccade to the direction of the sound after fixating a light. The magnitude of the reward delivered to the animal on correct trials was varied in some experiments. We also examined neural activity during an auditory task in which the animal was only required to fixate a light during sound presentation. We wanted to know whether the various nonauditory attributes of these tasks influence neural signals in the IC. 


\begin{tabular}{|c|c|c|c|c|}
\hline & \multicolumn{4}{|l|}{ Paradigm } \\
\hline & A, Saccade task & B, Variable reward saccade task & C, Fixation task & D, No task (sound only) \\
\hline Total cells & 95 & 52 & 26 & 25 \\
\hline Monkeys contributing & C and X & $C$ & $C$ & $C$ \\
\hline Light locations (degrees) & $-12,10,0,10$ and 12,10 & 0,10 & $-12,10,0,10$ and 12,10 & \\
\hline Light color & Green & Green or red ${ }^{a}$ & Red & \\
\hline Sound locations (degrees along horizontal meridian) & $\begin{array}{l}-23 \text { to } 23 \text { by } 5-6 \text { steps } \\
\text { ( } 9 \text { locations) }\end{array}$ & -12 and 12 & $\begin{array}{l}-23 \text { to } 23 \text { by } 5-6 \text { steps } \\
\text { ( } 9 \text { locations) }\end{array}$ & $\begin{array}{l}-23 \text { to } 23 \text { by } 5-6 \text { steps } \\
\text { (9 locations) }\end{array}$ \\
\hline Breakdown of individual neurons by paradigm & Monkey & $n$ & & \\
\hline A only & C & 45 & & \\
\hline A only & $x$ & 24 & & \\
\hline B only & $C$ & 27 & & \\
\hline$A$ and $C^{a}$ & $C$ & 26 & & \\
\hline$B$ and $D^{b}$ & $C$ & 25 & & \\
\hline
\end{tabular}

a Interleaved in four alternating blocks of $\sim 100$ trials each.

${ }^{b}$ Paradigm B was followed by $\sim 200$ trials of paradigm D.

\section{Materials and Methods}

General procedures. Two adult female rhesus monkeys (monkey C and monkey X) were subjects for these experiments. All animal procedures were conducted in accordance with the principles of laboratory animal care of the National Institutes of Health (publication 86-23, revised 1985) and were approved by the Institutional Animal Care and Use Committee at Dartmouth University. The monkeys underwent a surgery using isoflurane anesthesia and aseptic techniques to implant a head post for restraining the head and a scleral eye coil for monitoring eye position (Robinson, 1963; Judge et al., 1980).

The experiments were conducted in complete darkness in a singlewalled sound attenuation chamber (IAC, Bronx, NY) lined with soundabsorbent foam (3 inch painted SonexOne; Acoustical Solutions, Richmond, VA) to reduce echoes. The monkeys were seated comfortably in a conventional plastic primate chair (Crist Instruments, Hagerstown, MD) with a standard neck plate. The supports for immobilizing the head were located behind the interaural axis. The monkeys faced an array of lightemitting diodes (LEDs) and loudspeakers (Audax TWO25V2; Madisound Speaker Components, Madison, WI) located 57 inches away. The auditory stimulus was a band-limited white noise $(500 \mathrm{~Hz}$ to $18 \mathrm{kHz}, 10$ ms onset ramp, variable duration) at $50 \mathrm{~dB}$ sound pressure level (SPL) ("A" weighting; model 2237 integrating sound level meter with model 4137 condenser microphone; Brüel and Kjær, Naerum, Denmark) measured at the location normally occupied by the monkey's head. The input signal to each loudspeaker was adjusted to compensate for subtle differences between sound levels from each speaker ( $\pm 2 \mathrm{~dB}$ SPL). Eye position was sampled at $500 \mathrm{~Hz}$.

Experimental design. The experimental paradigms and stimulus conditions used in this study are summarized in Table 1 and Figure 1. The monkeys performed an auditory-guided saccade task (Fig. $1 A$ ), a visual fixation task while a sound was presented (Fig. $1 B$ ), or no task at all while a sound was presented (Fig. 1C). Training on these tasks has been described previously (Groh et al., 2003; Metzger et al., 2004).

In the saccade task (Fig. $1 A$ ), monkeys fixated a green LED at one of three randomly presented locations within the central $24^{\circ}\left(-12^{\circ}, 0^{\circ}, 12^{\circ}\right.$ horizontally and $10^{\circ}$ above the horizontal meridian). After a variable length of time (500-900 ms), the auditory stimulus was randomly presented from one of nine locations across the horizontal meridian $\left(-23^{\circ}\right.$ to $23^{\circ}$ in $5-6^{\circ}$ increments). The light remained on for another $500 \mathrm{~ms}$ (termed the "overlap" period) and then was extinguished, cuing the monkey to make a saccade toward the ongoing auditory target. The monkey was required to fixate the target for $\sim 500 \mathrm{~ms}$ to receive a liquid reward (i.e., reward was delivered exactly $500 \mathrm{~ms}$ after entry into the target acceptance window, so fixation may vary slightly from this). The sound remained on during this time. For trials in which the monkey did not accurately localize the target location, the target remained on for up to an additional $500 \mathrm{~ms}$, so that the monkey did not receive feedback regarding its accuracy until the trial ended (for example, if the saccade passed through the target acceptance window without stopping, the trial would not abruptly end before fixation occurred). The $10^{\circ}$ vertical separation between the fixation lights and auditory targets ensured that a saccade was required for all fixation-target combinations. Target acceptance windows were $8^{\circ}$ or $11^{\circ}$ in radius depending on target distance from the fixation light; the fixation light was never within the target acceptance window. All properly initiated trials (i.e., trials in which the monkeys maintained their gaze at the fixation light until its offset) were included for data analysis whether rewarded or unrewarded, provided a saccade (with any direction) began $>100$ and $<500 \mathrm{~ms}$ after the offset of the fixation light, and the endpoint was within the appropriate vertical range of $\pm 11^{\circ}$. These criteria helped ensure that the monkey was attentive and focused on the sound location range. Saccade onset was defined as the time when eye-movement velocity exceeded $20 \%$ s (velocity had to remain above this threshold for $20 \mathrm{~ms}$ to be considered a saccade), and offset was the time when velocity fell below $20 \%$ (for a minimum of 10 $\mathrm{ms}$ ). With these inclusion criteria, $90.3 \%$ of all properly initiated trials were included in the final analysis of the saccade task. Of these, $76.9 \%$ were rewarded trials.

The "variable-reward saccade" task was a variant of the saccade task and was presented in separate experimental sessions. In this task, the magnitude of the reward varied on different, interleaved blocks of $\sim 100$ trials. Reward size was signaled to the monkey by the color of the fixation light. When the fixation light was green, the monkey received two drops of liquid reward for a correct trial. When the fixation light was red, only one drop was delivered after a correct trial. For this task, only one fixation location, at $0^{\circ}$ horizontal and $10^{\circ}$ vertical, was used. The sound was presented randomly from one of two locations $\left( \pm 12^{\circ}\right.$ on the horizontal meridian).

In other experimental sessions, 26 neurons were tested during both the saccade task and a fixation task (Fig. $1 B$ ). The two tasks were interleaved in four alternating blocks of $\sim 100$ trials each. The fixation task began exactly as the saccade task, except the fixation LED was red. The two tasks diverged at the end of the overlap period (i.e., $500 \mathrm{~ms}$ after sound onset); in the fixation task, the light remained on with the sound for another 800 ms for continued fixation. To receive a liquid reward, the monkey was required to maintain fixation of the light throughout the trial. To further ensure that we assessed only trials in which the monkey knew which task was being presented, we discarded the first 10 trials of each block. For other experiments in which only one task was presented, the task was presented for several trials before recording the cell.

For 25 of the 52 experimental sessions in which the variable-reward saccade task was presented, a block of $\sim 200$ trials was presented at the end of the session in which each trial consisted of a sound presentation only (i.e., no behavioral task imposed nor reward administered) (Fig. 1C).

Recording. Once the animals were trained, a cylinder was implanted using stereotaxic techniques in an aseptic surgical procedure under 
A

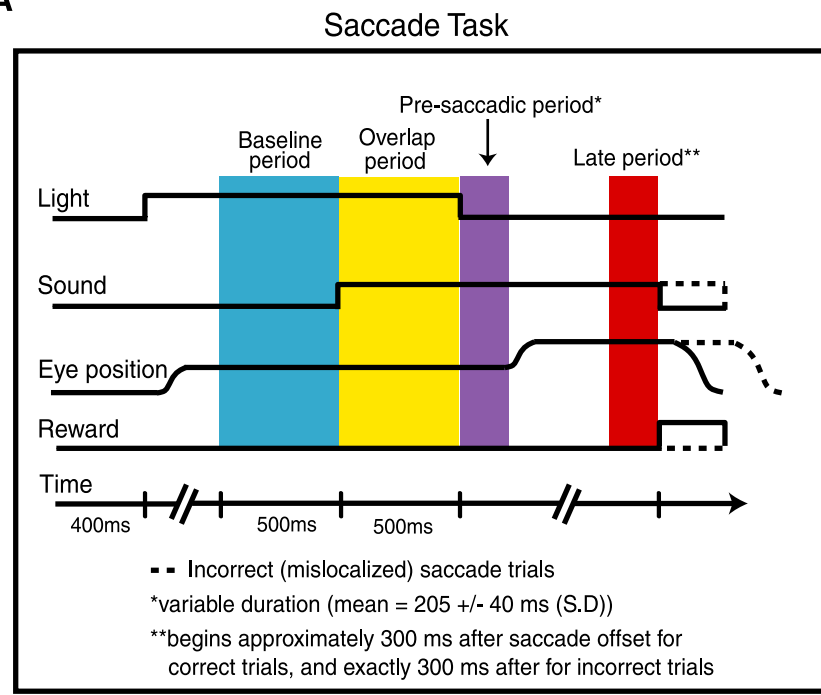

B

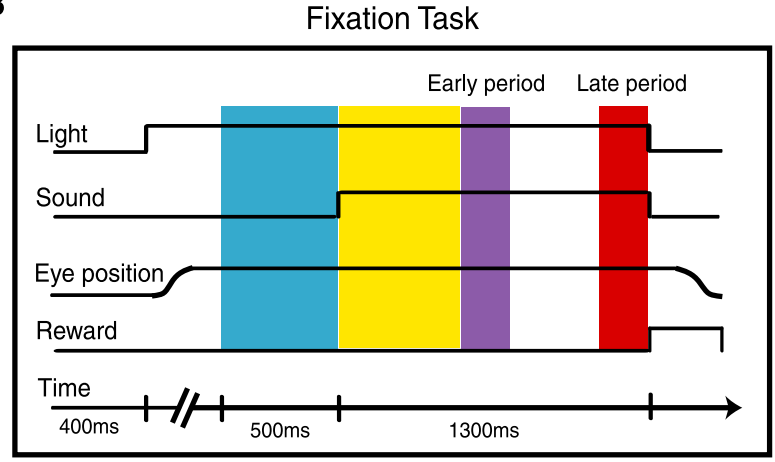

C

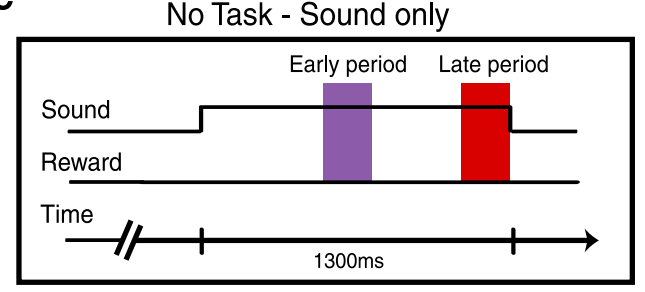

Figure 1. Schematic representation of the time course of the auditory saccade task $(\boldsymbol{A})$, the fixation task $(\boldsymbol{B})$, and a paradigm in which only a sound was presented (i.e., no task was imposed). Colored bars indicate the designated time periods chosen for statistical analysis in each paradigm. Upward steps in the traces for light, sound, and reward indicate presentation of that stimulus. Vertical deflections in the eye-position trace indicate a saccade (of any direction and amplitude).

isoflurane anesthesia. The cylinder was placed over one side of the skull such that electrodes approached the ipsilateral IC at an angle of $33^{\circ}$ lateral from vertical in the coronal plane. Search stimuli consisted of broadband noise randomly presented from any of the speaker positions used for that experiment (Table 1). Once the electrode had reached the IC (as determined by the advent of acoustic responses in the multiunit activity), any neuron with isolatable action potentials was accepted for recording purposes. Standard recording techniques were used: a varnish-coated tungsten electrode ( $\sim 2 \mathrm{M} \Omega$; Frederick Haer Company, Bowdoinham, ME) was advanced with a hydraulic micropositioner (Narishige, Tokyo, Japan). Electrical potentials were amplified, and action potentials were detected using either a dual-window discriminator (Bak Electronics, Mount Airy, MD) or a template-matching spike-sorting method (Alpha Omega Engineering, Nazareth, Israel). The time of occurrence of action potentials was stored for off-line analysis.
If time and unit isolation allowed, neurons ( $n=58$ total) were tested for sound frequency sensitivity after the presentation of the experimental $\operatorname{task}(\mathrm{s})$. Tones (500 ms at $50 \mathrm{~dB}$ SPL) were presented randomly from a range of $400 \mathrm{~Hz}$ to $12 \mathrm{kHz}$ (usually in $\sim 1 / 3$ octave increments) from a single contralateral sound location. The tones were presented in either the fixation task (for monkey X) or no task at all (for monkey C). For each neuron, breadth of tuning was assessed by evaluating the auditory response at each frequency using a two-tailed paired $t$ test (comparing the average discharge rate for the first $500 \mathrm{~ms}$ after sound onset with the average discharge rate of the baseline period, with $p$ values Bonferronicorrected according to the number of tested frequencies). Neurons were classified as responsive only to low frequencies ( $\leq 1000 \mathrm{~Hz} ; 20$ neurons), responsive to both low and high ( $\geq 2000 \mathrm{~Hz}$ ) frequencies ( 27 neurons), or not responsive to any individual frequency (10 neurons). Neurons responsive only to high frequencies were too rare (one neuron) to include as a separate category for analysis. This bias for low frequencies and/or broad tuning is consistent with our previous studies in primate IC (Groh et al., 2003; Porter et al., 2006). As we noted previously (Porter et al., 2006), a possible explanation for this pattern comes from various studies that have found asymmetries in frequency tuning curves: the cutoff for high frequencies tends to be sharper than that for low frequencies (mouse IC, Egorova et al., 2001; Hage and Ehret, 2003; Yan et al., 2005; cat primary auditory cortex, Sutter, 2000; mouse auditory nerve fibers, Taberner and Liberman, 2005). Low-frequency sounds at a fixed, suprathreshold intensity will therefore activate many neurons with these "low-frequency tails," whereas high-frequency sounds at that same intensity will not. Another possibility is that the primate IC simply has an expanded representation of low-frequency sounds. Most of the characteristic frequencies reported in primate IC by Ryan and Miller (1978) were below $2 \mathrm{kHz}$ ( 25 of 27 neurons).

Recording locations. The locations of our recording penetrations were identified by using magnetic resonance imaging (MRI) at the Dartmouth Brain Imaging Center [GE 1.5T scanner (GE Healthcare, Little Chalfont, UK); three-dimensional T1-weighted gradient echo pulse sequence; 5 inch receive-only surface coil]. Several tungsten electrodes were inserted into the brain for the scan; these electrodes were readily visible in the images and served as reference points for the reconstruction of the recording locations as viewed and measured in three dimensions using SPM'99 (The Wellcome Trust, London, UK). Although the IC was readily apparent in the MR images, finer distinctions of the $\mathrm{IC}$, such as the subnuclei of the IC, were not. Recordings were made from the left IC of monkey $\mathrm{C}$ and the right IC of monkey $\mathrm{X}$.

In monkey $\mathrm{X}$, the recording site locations were confirmed histologically as well as by MRI (courtesy of Jeffrey A. Winer and David T. Larue, University of California, Berkeley, CA) (Porter et al., 2006). Standard histological techniques were used: the brain was fixed with Formalin and sliced in $50 \mu \mathrm{m}$ sections that were stained with cresyl violet. Because of the long period of time over which the recordings took place, we did not attempt to assign specific individual sites to the IC subdivisions identified in other species.

Data analysis. Baseline neural activity was assessed during a $500 \mathrm{~ms}$ period before the onset of the auditory stimulus in each trial (Fig. 1). Neurons were considered auditory if their discharge rate during the overlap period differed significantly from baseline activity (two-tailed paired $t$ test, $p<0.05$ ). Only auditory neurons were used for subsequent analyses. Because none of the auditory neurons exhibited a significantly lower discharge rate for the entire overlap period, our analyses included only excitatory auditory neurons. Spikes were also counted during time windows specific to each task (Fig. 1) to statistically evaluate neural activity at later time points in the tasks. For the saccade task, the "late" period was designated as the last $200 \mathrm{~ms}$ of the trial for correct trials. This was also the last $200 \mathrm{~ms}$ of target fixation and coincided closely with 300-500 ms after saccade offset. For incorrect trials, a corresponding time period was demarcated using saccade timing (because fixation was outside the target acceptance window, there was no entry time into the acceptance window to be measured). Thus, the late period was designated as exactly 300-500 ms after saccade offset. Note that, for incorrect trials, the late period did not end with the trial; the sound presentation (and trial) could last up to $\sim 500 \mathrm{~ms}$ longer (see above). For all trials, the 
A

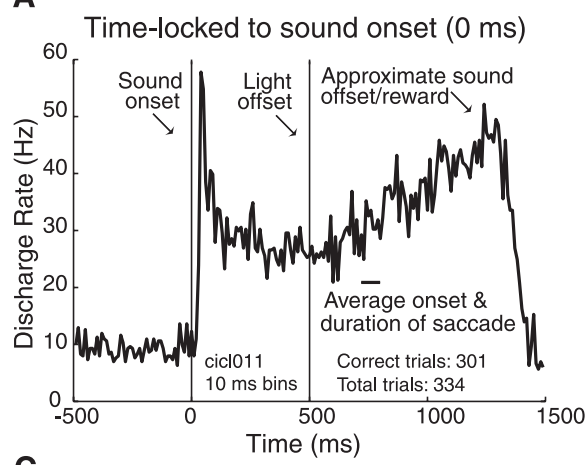

C

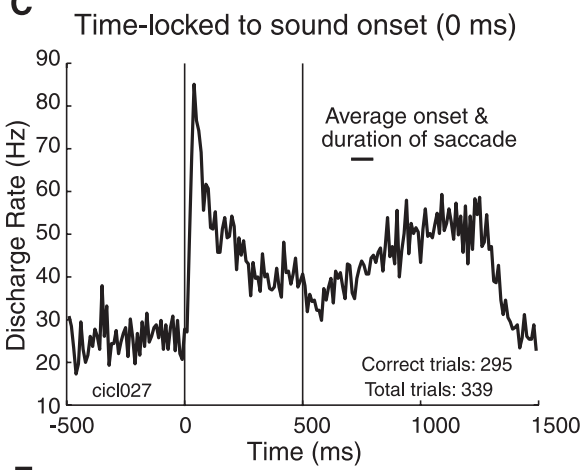

E Time-locked to sound onset $(0 \mathrm{~ms})$

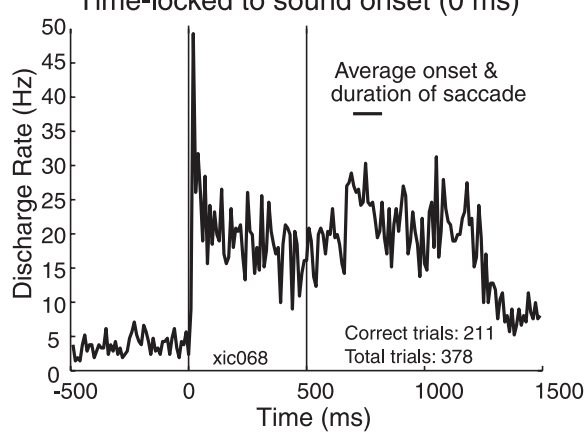

B

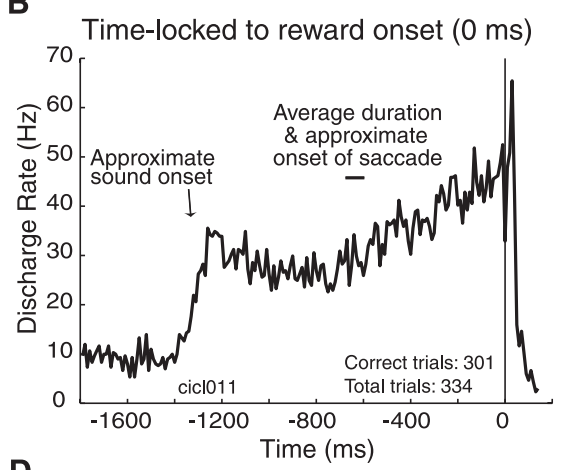

D

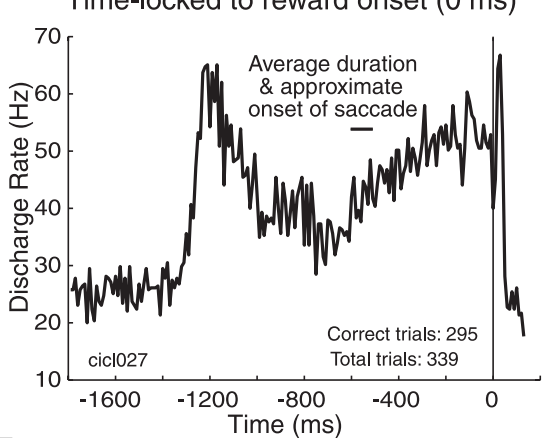

$\mathbf{F}$

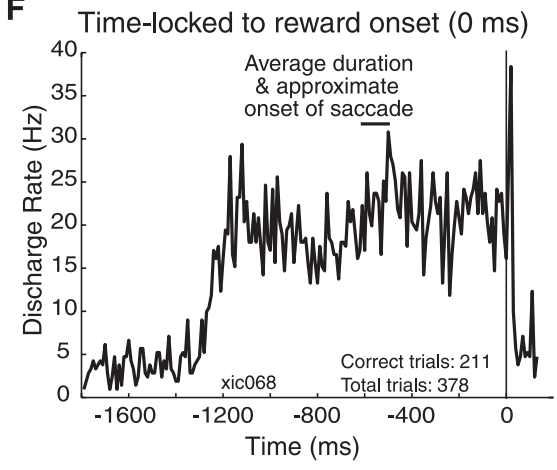

Figure 2. Neural activity of three representative neurons that exhibited late-trial activity during the saccade task. A, C, E, PSTHS of discharge rate in three neurons time locked to sound onset. Note that the end of the trial (sound offset) does not necessarily occur at the same time for all trials (attributable to variations in the timing of saccade execution). B, D, F, PSTHs of discharge rate in the same three neurons as $A, C$, and $\boldsymbol{E}$ but time locked to reward onset. Note that only correct trials are shown in $A, C$, and $E$ as well, for comparison with $\boldsymbol{B}, \boldsymbol{C}$, and $\boldsymbol{F}$ (although incorrect trials were included for all statistical analyses). The saccade period varied in duration across trials with a mean $\pm S D$ of $95 \pm 43 \mathrm{~ms}$ in $\boldsymbol{A}$ and $\boldsymbol{B}, 94 \pm 41 \mathrm{~ms}$ in $\boldsymbol{C}$ and $\boldsymbol{D}$, and $125 \pm 46 \mathrm{~ms}$ in $\boldsymbol{E}$ and $\boldsymbol{F}$ (values include incorrect trials).

"presaccadic" period was the interval from light offset to saccade onset (mean duration, $205 \pm 40 \mathrm{~ms}$ ).

\section{Results}

We found that the neural activity of many IC neurons had intriguing attributes during an auditory saccade task. These neurons displayed a gradual increase in discharge rate late in the time course of these trials that appeared to reflect anticipation of the reward. Figure 2 shows several individual examples of neurons that exhibited this late-trial activity during the saccade task. The peristimulus time histograms (PSTHs) show that the discharge rate gradually increased from approximately the time of light offset, continuing through the period of target fixation, until the end of the trial. The PSTHs are aligned on sound onset/light offset in the left column (Fig. 2A,C,E) and on reward onset/sound offset in the right column (Fig. $2 B, D, F$ ). [The interval between light offset and reward onset was variable depending on the length of time it took the animal to get its eyes into the reinforcement window. The increase in activity begins to occur at approximately the time of light offset (time $=500 \mathrm{~ms}$; left panels) and peaks at approximately the time of the sound offset and reward (time $=0 \mathrm{~ms}$; right panels).]

To statistically evaluate the late-trial activity in the saccade task, we compared the discharge rate in two time periods of interest: a late period and a presaccadic period (Fig. 1A) (see Materials and Methods). Both the late period and the presaccadic period shared similar characteristics in that the sound was on, the light was off, and the eyes were stationary. If the discharge rate during the late period was greater than that of the presaccadic period (one-tailed paired $t$ test, $p<0.025$ ), then the neuron was considered to have latetrial activity. This was the case for 55 of 95 neurons tested (58\%; 44 of 71 from monkey C and 11 of 24 from monkey X) in the saccade task. Figure 3 shows a population PSTH of these cells time locked to sound onset/light offset (Fig. $3 A$ ), as well as time locked to reward onset (Fig. $3 B$ ).

The eyes were in different positions during these two time periods, so in principle, this test could have picked up neurons based on their eye-position sensitivity (Groh et al., 2001; Zwiers et al., 2004). However, this test was conducted on pooled data involving both leftward and rightward saccades to sounds, which would have tended to minimize the contribution of eye-position sensitivity to the population of neurons selected by this means. To examine this, we tested (onetailed paired $t$ test, $p<0.025$ ) for late-trial activity in trials with contralateral saccades separately from trials in which the saccade was in an ipsilateral direction. Representation of eye position in primate IC neurons has been found to be predominately contralateral (Porter et al., 2006), so moving the eyes contralaterally should generally increase neural activity, thus making it appear that late-trial activity is more prominent in these trials than trials in which the saccade was in an ipsilateral direction. However, the proportion of neurons showing late-trial activity was similar when the data were broken down in this manner: we found that $58 \%$ of cells had significant late-trial activity when assessing trials with contralateral saccades versus $51 \%$ of cells when assessing trials with ipsilateral saccades. Moreover, cells that had significant late-trial activity for contralateral saccades also tended to have this activity for ipsilateral saccades (Fisher's exact test on an association between the two proportions of cells, $p<0.025$ ). This suggests that changing eye position had little to no effect on the late-trial activity.

To examine whether the increase in activity late in the trial reflects reward anticipation, we varied the magnitude of the reward. That the monkey detected the difference in reward value 
A

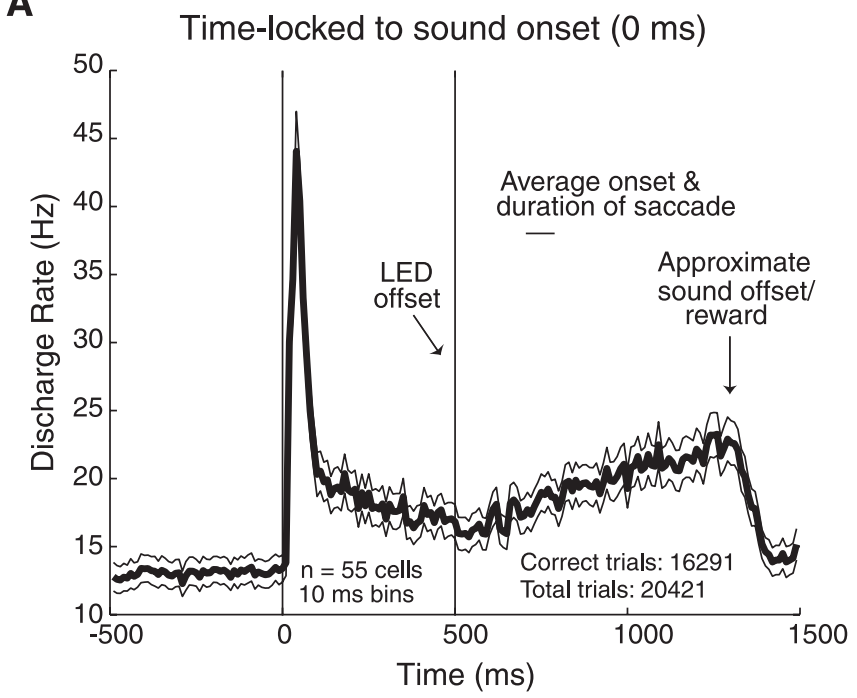

B

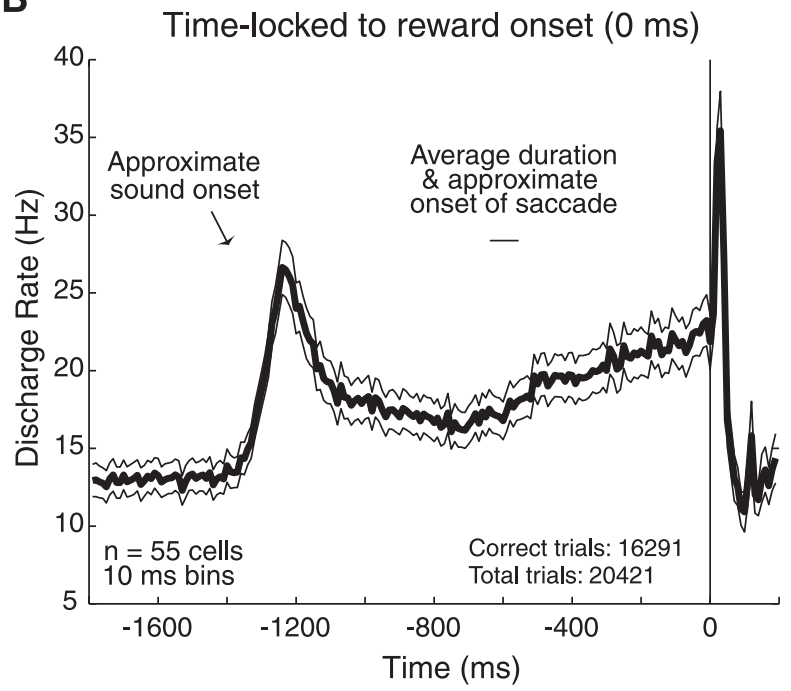

Figure 3. Neural activity of the population of neurons that had significant late-trial activity during the saccade task. $A$, PSTH of discharge rate (with SES) in which trials were time locked to sound onset. $\boldsymbol{B}$, PSTH of discharge rate (with SES) in which trials were time locked to reward onset. Note that only correct trials are shown in $\boldsymbol{A}$ as well, for comparison with $\boldsymbol{B}$ (although incorrect trials were included for all statistical analyses). The saccade period varied in duration across trials with a mean of $84 \pm 27 \mathrm{~ms}$ (including incorrect trials).

could be seen in its behavior: reaction times were longer in the low-reward trials (one-tailed unpaired $t$ test, $p<0.025$ ), to both initiate trials (via the saccade to the fixation light) and complete trials (via the saccade to the sound) (Fig. 4A).

We recorded the activity of an additional 52 neurons during performance of this variable-reward task. An effect of reward magnitude was evident not just during the late period but also during earlier time periods, including the baseline period. Figure $4 B$ shows the difference in neural activity between high- and low-reward trials for the population of neurons when comparing the average activity during four time periods: baseline, the last $200 \mathrm{~ms}$ of overlap, presaccade, and late. Whether assessing each time period individually or combining all four periods, the average discharge rate in the high-reward trials was significantly greater than in the low-reward trials (one-tailed $t$ test on the average difference in discharge rate vs 0 , Bonferroni-corrected, $p<0.025)$. Figure $4 D$ shows a composite of three population
PSTHs in which each PSTH was constructed from those neurons in which the activity during high-reward trials was significantly greater than low-reward trials for the corresponding time period (baseline, last $200 \mathrm{~ms}$ of overlap, or late period; one-tailed unpaired $t$ test, Bonferroni-corrected, $p<0.025$ ).

The use of a blocked design could have impacted the results of the manipulation of reward magnitude, because drift in uninteresting factors such as the quality of unit isolation could have produced differences in the level of activity across blocks. However, several aspects of the experimental design and results mitigate this concern. (1) Between four and eight blocks were run in alternation, so that slow changes in unit properties unrelated to the task would not tend to be correlated with one type of block versus another. (2) The observed effect was generally an increase in responsiveness on high-reward magnitude blocks rather than a change in responsiveness that could be in either direction. (3) To determine whether an effect of reward magnitude remained after any nonstationarities were accounted for, we conducted a twoway ANOVA with block number and reward size as the two factors and the activity during the baseline period as the dependent variable. We found a significant main effect for reward size or interaction term in 35 of 52 neurons [ $67 \%$; interaction terms, $n=$ 17; main effect for reward (interaction term not significant), $n=$ $18 ; p<0.025$ ], indicating that reward size exerted a statistically significant influence on neural activity in the majority of neurons even when block number was accounted for. Indeed, controlling for block number increased the proportion of neurons showing a statistically significant affect of reward size (compared with the results using a $t$ test) (Fig. $4 D$ ).

Block number also affected activity, as can be seen from the neurons with significant interaction terms, as well as an additional 16 neurons that showed a main effect for block size (seven of these neurons also were among those with a main effect for reward size). This finding is consistent with our hypothesis that IC neurons contain reward-related signals: the animal's motivation likely fluctuates during the course of the experimental session, so variation in background activity levels across blocks is actually to be expected, although of course it is difficult to prove that such variation is related to reward and not to uninteresting factors such as fluctuations in unit isolation.

Because the late-trial activity in the saccade task may be associated with reward anticipation, we compared this activity in trials that followed correct (rewarded) trials versus trials that followed incorrect (not rewarded) trials in the population of cells that are significant for late-trial activity in the saccade task. The population PSTHs (time-locked to saccade onset) for the two conditions were virtually identical (Fig. 5A), suggesting that reward status or performance in the previous trial does not influence the late-trial activity in the following trial. We also compared the late-trial activity in correct trials versus incorrect trials and observed no notable differences in the population PSTHs for these two conditions (Fig. 5B), suggesting that the monkey's ultimate success at localizing the target does not influence the latetrial activity in the same trial.

We wondered whether performance of a sound localization task was a necessary condition for observing late-trial activity or if the same activity might be observed in a rewarded task in which the sound was behaviorally irrelevant. Accordingly, we tested an additional 26 neurons on both a visual fixation task (in which sounds were to be ignored) (Fig. $1 B$ ) and the saccade task. Acoustic parameters in the fixation task were matched to the saccade task, including the sound duration $(1300 \mathrm{~ms})$, which closely matched the average sound duration during the saccade task 
A

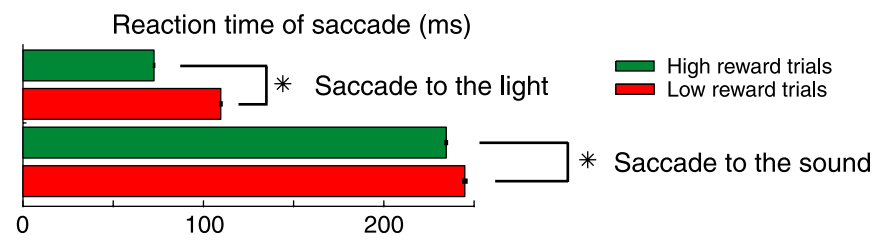

B

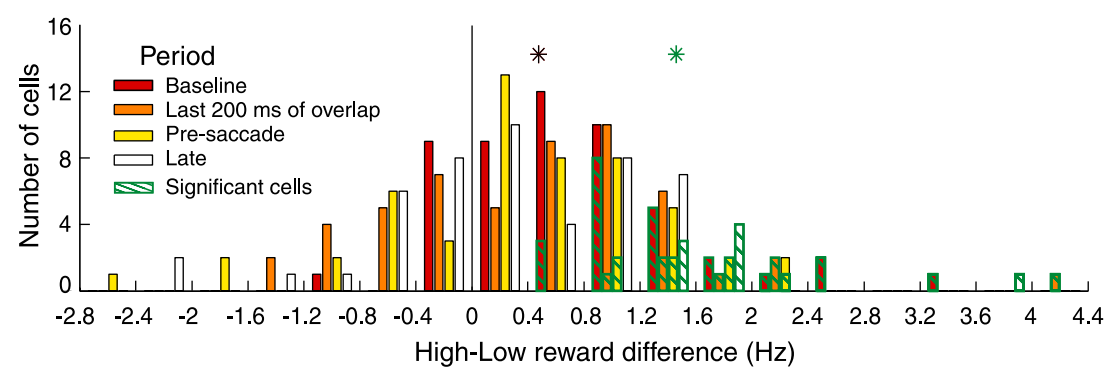

C

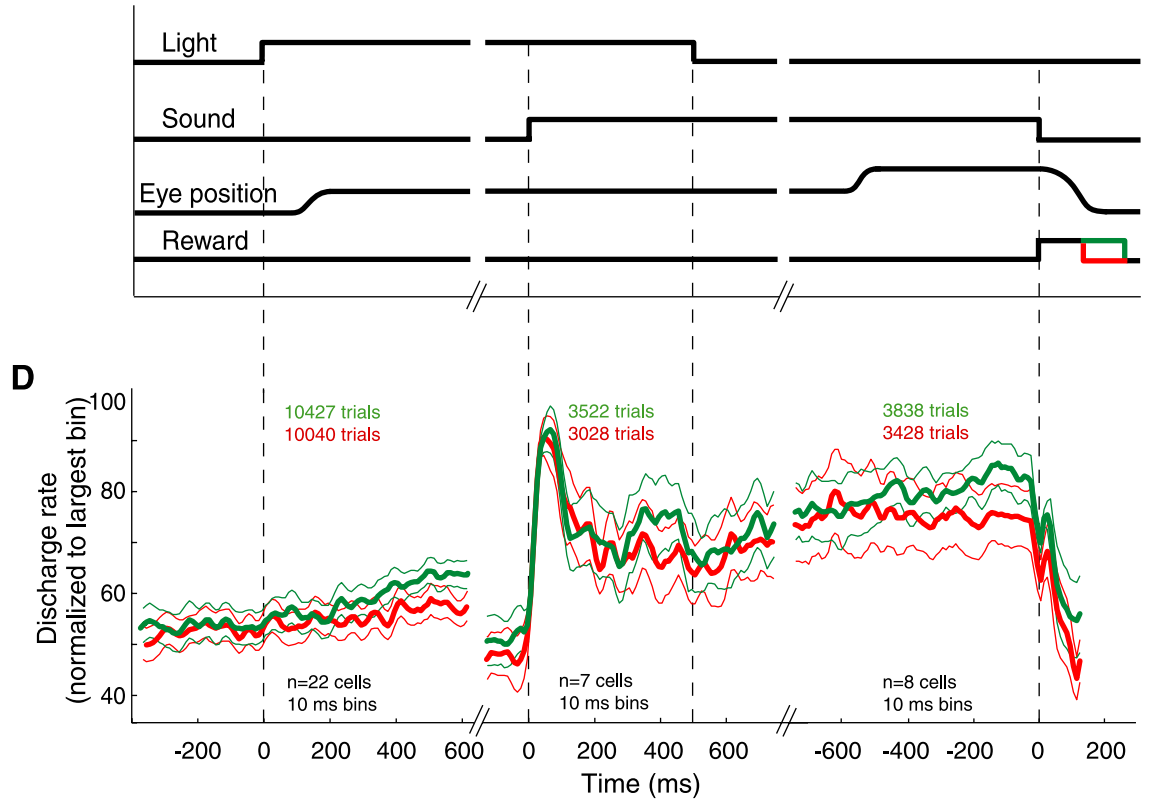

Figure 4. Behavioral and physiological differences observed during the high- and low-reward trials of the variable-reward saccade task. $A$, Histogram of saccade reaction times [the interval between onset of the light (or onset of the sound) and the entrance of the monkeys eyes into the corresponding target acceptance window, with SES]. Asterisks indicate statistical significance (one-tailed unpaired $t$ test, $p<0.025$ ). $\boldsymbol{B}$, Histogram of the difference in average discharge rate for each period in the high-reward trials versus the low-reward trials. For all 52 neurons, the mean of these differences for each time period was significantly different from 0 (one-tailed $t$ test, Bonferroni-corrected, $p<0.025$ ), as well as when all four time periods were combined (black asterisk). Green hatches indicate those neurons in which the discharge rate in the high-reward trials was greater than in the low-reward trials for at least one time period (one-tailed unpaired $t$ test, Bonferroni-corrected, $p<0.025$ ), and the green asterisk indicates the mean of the average differences across all four periods for this subpopulation. $C$, Schematic representation of the time course of events during the saccade task. $\boldsymbol{D}$, Reward-modulated activity during the saccade task. Three PSTHs (with SEs) time locked at $0 \mathrm{~ms}$ to light onset (left), sound onset (middle), or reward onset (right) for the subpopulation of cells in which the activity during the high-reward trials (green) was significantly greater than that of the low-reward trials (red) for the corresponding time period [baseline (left), last $200 \mathrm{~ms}$ of overlap (middle), and late (right); one-tailed unpaired $t$ test, Bonferronicorrected, $p<0.025]$. The traces were smoothed by convolving with a filter with points $(0.11,0.22,0.33,0.22,0.11)$ across adjacent bins. Note that only correct trials are shown in all three PSTHs (for comparison with the PSTH time locked to reward onset), although incorrect trials were included for all statistical analyses.

(mean $\pm \mathrm{SD}, 1284 \pm 36 \mathrm{~ms}$ ). The late period in the fixation task was designated as the last $200 \mathrm{~ms}$ of the trial (like in the saccade task) and compared with an earlier time interval of 500-700 ms after sound onset (i.e., corresponding closely to the timing and duration of the presaccadic period of the saccade task). If the discharge rate during the late period was greater than the early time period (according to a one-tailed paired $t$ test, $p<0.025$ ), then the neuron was considered to have late-trial activity. This was the case for 10 of 26 cells (39\%) tested in the fixation task. Figure 6 (red) shows a population PSTH of these cells, in which a gradual increase in discharge rate at the end of the task is readily apparent. Note that the duration between sound onset and light offset/reward delivery is fixed. Thus, the PSTH is time locked to both events. [The abovebaseline activity at reward onset (after trial end) may be a response to the positive reinforcement, a sensory response attributable to the sound of the reward delivery mechanism (i.e., solenoid activation) and/or other sounds associated with liquid reward delivery (e.g., licking), or electrical artifact caused by the contact of the animal's tongue with the metal juice tube. Because we could not rule out uninteresting sensory or artifactual components to this activity, we did not analyze it further.]

During examination of neural activity in these 26 cells during the saccade task, we found that the same 10 neurons were significant for late-trial activity in the saccade task as well, although three additional neurons were significant in the saccade task (making a total 13 of 26 cells; $50 \%$ ). Figure 6 compares the late-trial activity in the fixation task with that observed in the saccade task for all 10 cells that had significant late-trial activity in both tasks. It appears that the late-trial activity is similarly robust and follows a similar time course in both tasks. Indeed, a cell-by-cell comparison of the mean discharge rate during the late period in the saccade task versus the fixation task (two-tailed unpaired $t$ test, $p<0.05$, after subtraction of the presaccade or early period, respectively) reveals no difference in magnitude of the late-trial activity for 7 of the 10 cells. These results therefore suggest that the specific requirement to saccade to the sound is not necessary to elicit the late-trial ramping activity.

In 25 of the 52 experimental sessions in which the variable-reward saccade task was presented, this paradigm was followed by a block of trials in which only a sound was presented (1300 ms band-limited white noise), with no behavioral task imposed or reward administered (Fig. 1C). Of these 25 neurons, nine were found to have statistically significant late-trial activity during the variable-reward saccade task, but none had late-trial activity during the sound-only paradigm (one-tailed paired $t$ test, $p<0.025$, comparing the late period with the early time period, as in the fixation task), suggesting that the late-trial activity observed during the saccade task is reward related and/or influenced by behavioral activity.

In examining sound location sensitivity (with respect to the head) during the saccade task, we found that neural activity could 
A

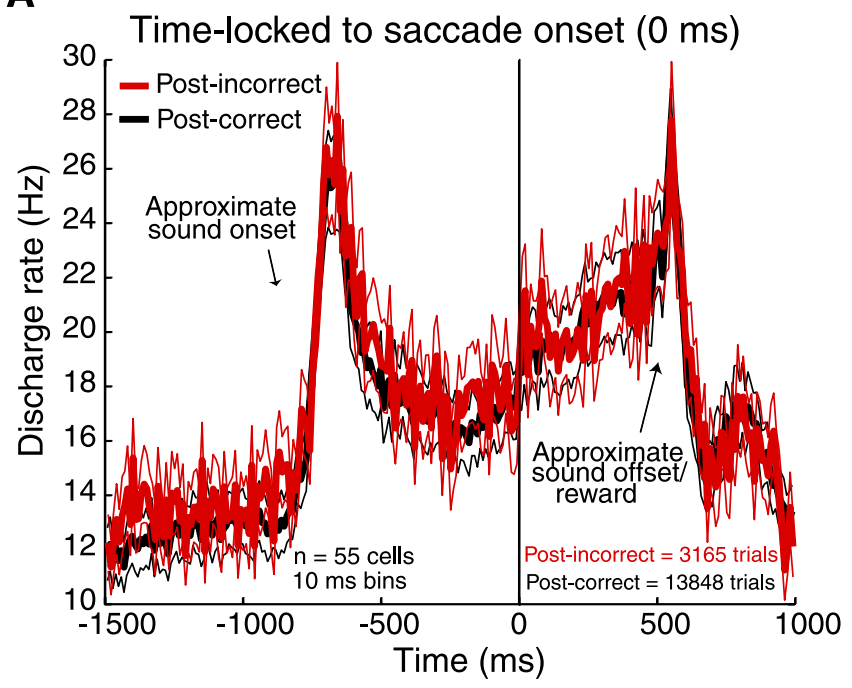

B

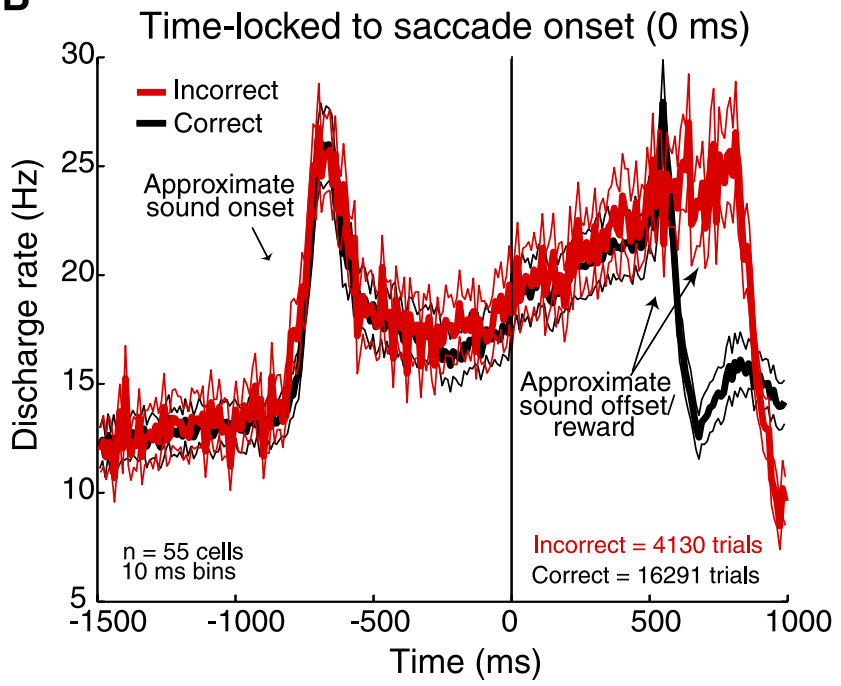

Figure 5. Reward/success status and late-trial activity in the population of neurons that had significant late-trial activity during the saccade task. $\boldsymbol{A}$, PSTHs of discharge rate (with SEs) for those trials that followed an incorrect trial (red) and for those trials that followed a correct trial (black). Note that not all trials fit for analysis in this study are included in these PSTHs because some were not immediately preceded by a fit trial. For example, if a trial was excluded for analysis in this study attributable to reasons described in Materials and Methods, then the next fit trial was not used in either PSTH here. Thus, 3408 of 20,421 trials were excluded in preparation of these PSTHs. B, PSTH of discharge rate (with SEs) for incorrect trials (red) and for correct trials (black).

vary with sound location during any part of the trial. Figure 7 shows the neural activity of a neuron (the same neuron shown in Fig. $2 C, D$ ) during the saccade task as a function of sound location. Like many neurons, this neuron displayed a decrease in overall responsiveness to ipsilateral sounds, although the latetrial activity was still visible for all three sound location ranges. For cells that had statistically significant late-trial activity, spatial sensitivity during the late period (as assessed by one-way ANOVA on effect of sound location across all nine individual locations on discharge rate, $p<0.05$ ) was slightly less common ( 21 of 55 cells) than that observed in the initial auditory response (overlap period; 30 of 55 cells). Also, spatial sensitivity in the late period was not associated with the spatial sensitivity in the overlap period (two-tailed Fisher's exact test on the proportion of cells with

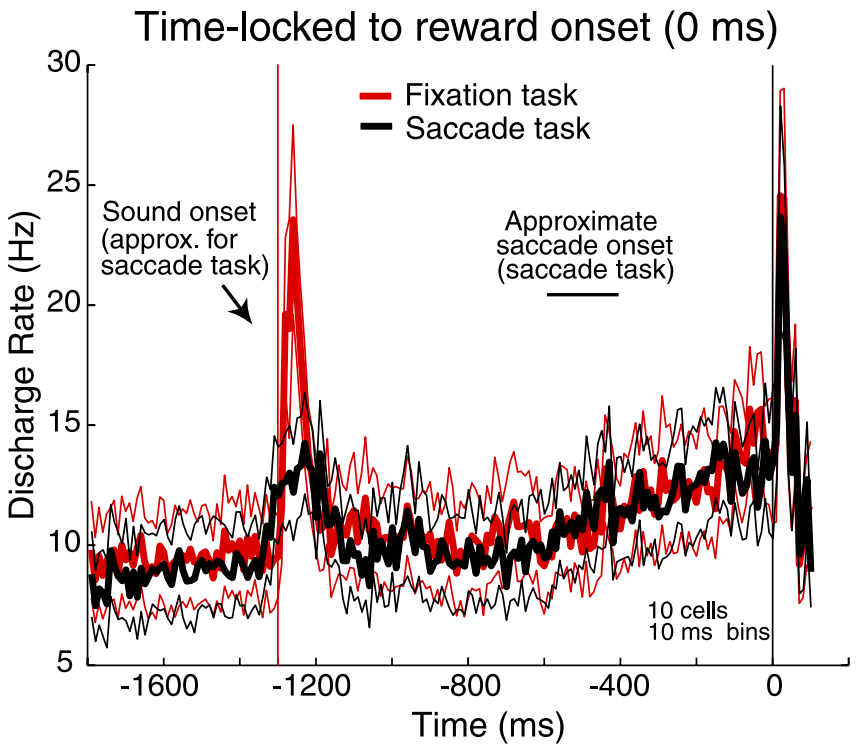

Figure 6. Two population PSTHs of the discharge rate (with SES) of the neurons that had significant late-trial activity during the saccade task (black) and the fixation task (red). In both cases, the trials are time locked to reward onset to allow for easier comparison of the late-trial activity. Thus, for the saccade task PSTH, only correct trials are shown (3121 correct trials of 3779 trials evaluated statistically). Note that, for the fixation task, sound offset, light offset, and reward delivery occur simultaneously. Thus, the PSTH is time locked to this time point as well as sound onset. Also note that, unlike the fixation task PSTH, sound onset does not occur at the same time in all of the trials for the saccade task PSTH because of variability in saccade execution timing.

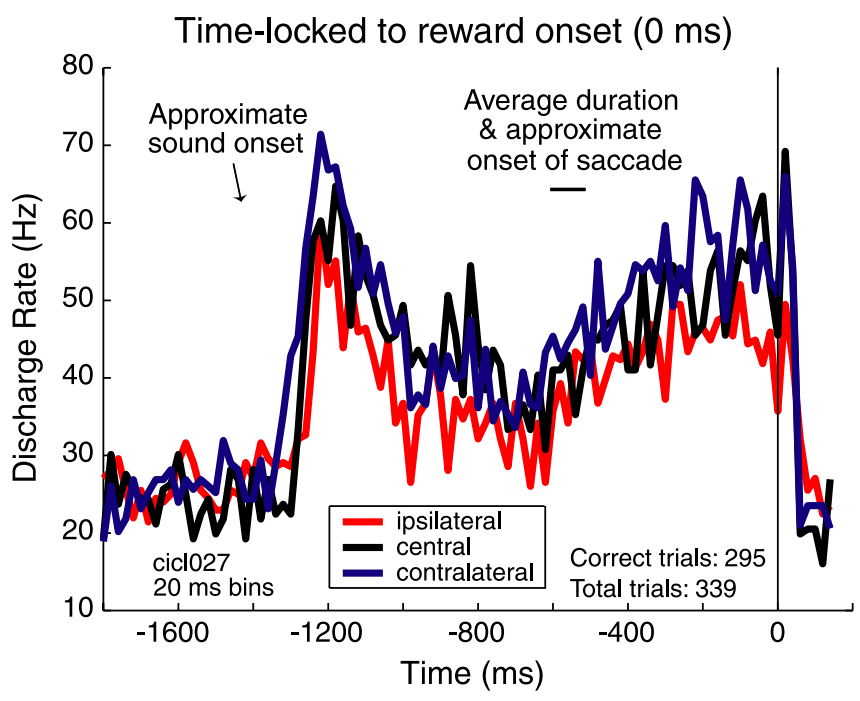

Figure 7. Neural activity of the same neuron shown in Figure 2, $\mathbf{C}$ and $\mathbf{D}$. Three PSTHs were prepared by averaging the discharge rate in trials in which the sound was presented from the three contralateral locations (blue), the central three locations (black), or the three ipsilateral locations (red). All three PSTHs were time locked to reward onset (thus, only correct trials are shown, although incorrect trials were included for all statistical analyses). The saccade period varied in duration across trials with a mean of $94 \pm 41 \mathrm{~ms}$ (including incorrect trials).

late-trial activity that displayed spatial sensitivity during both periods, $p<0.05$ ), suggesting that the late-trial activity is less a function of acoustic spatial parameters. However, of all 95 cells tested, those that show spatial sensitivity in the initial auditory response are more likely to exhibit significant late-trial activity (two-tailed Fisher's exact test, $p<0.025$ ).

In examining the class of frequency sensitivity for neurons (low, broad tuning, or neither; see Materials and Methods), 27 of 
58 neurons were broadly tuned to tone frequency, 20 were low, and 10 were neither. In evaluating both frequency sensitivity class and late-trial activity in the saccade task, $\chi^{2}$ analysis did not reveal any statistically significant association between late-trial activity and the three categories of frequency tuning, suggesting that frequency sensitivity was uncorrelated with the presence of late-trial activity.

\section{Discussion}

Results presented in this study offer novel evidence for the existence of a nonauditory signal in the primate IC that influences neural activity during two disparate behavioral tasks. In the saccade task, most (58\%) neurons displayed a gradual increase in activity in the latter part of the trials, although the auditory stimulus remained constant during this period. Because a reward was delivered at the end of most trials (i.e., $77 \%$ of the saccade task trials were judged correct), this "ramping" activity resembled an anticipatory signal for reward (or "reward expectation" signal) (Schultz, 2000). Although the late-trial activity was observed during incorrect (not rewarded) trials, this may be attributable to an inability of the monkeys to discern the likelihood of reward, because their behavior was similar to that observed during correct trials [i.e., they quickly (within $500 \mathrm{~ms}$ ) made a saccade to, and fixated, a location along the horizontal meridian]. In addition, the incorrect trials may not have occurred frequently (or consistently) enough to allow for extinction of an association between sound and reward. In fact, in the 25 experimental sessions in which the variable-reward saccade task was followed by a block of trials of the sound-only paradigm (no responses required or reward delivered), none of the neurons were found to have statistically significant late-trial activity in the sound-only paradigm. This further suggests that the late-trial activity is reward related and may be extinguished in the prolonged absence of reward, although it cannot be ruled out that some concurrent behavioral activity is required.

Recently, Komura et al. (2001, 2005) observed similar activity in the rat auditory thalamus (i.e., a gradual increase in neural activity during trials that terminated with the trial). In addition, they demonstrated that this "late response" could be modulated by reward value. We also found evidence suggesting that IC activity can be modulated by reward magnitude. Specifically, when the color of the fixation light signaled a larger impending reward, the discharge rate in all time periods tended to be greater than that observed in trials in which the fixation light signaled the smaller reward volume.

Although it is unclear how reward-related signals may reach the IC, the SC and the substantia nigra (SN) are two structures that project to the IC (SC, Harting, 1977; Adams, 1980; Doubell et al., 2000; Winer et al., 2002; SN, Adams, 1980; Coleman and Clerici, 1987; Olazabal and Moore, 1989; Yasui et al., 1991; Moriizumi et al., 1992; Winer et al., 2002) and exhibit activity related to reward expectation (SC, Ikeda and Hikosaka, 2003; SN, Schultz et al., 1997; Sato and Hikosaka, 2002; Satoh et al., 2003), although it is unlikely that the dopaminergic neurons in the SN (which encode reward prediction error) project directly to the IC (Yasui et al., 1991; Moriizumi et al., 1992). Another possibility exists with descending connections within the auditory pathway. Reward-related activity that was observed in thalamic neurons (Komura et al., 2001, 2005) may be transmitted to the IC (Adams, 1980; Kuwabara and Zook, 2000; Winer et al., 2002). Additional task-related signals have been reported in auditory cortex (Durif et al., 2003; Brosch et al., 2005); these too could be transmitted to the IC via descending projections (Huffman and Henson, 1990).
Whether the reward-related signal also reflects other cognitive influences, such as increased attention to the sound, is not known. Changes in attention appear to occur in the variablereward saccade task because the reaction times are different in the high- and low-reward trials. However, it seems unlikely that attention is the sole cause of the activity patterns we observed because the magnitude of the late-trial activity was similar in the saccade and fixation tasks, although it would be expected that more attention would be allocated to the sound in the saccade task. It should be noted, however, that other characteristics of the auditory stimuli (such as timing) may be important for this effect and similarly attended by the monkey across tasks. Because the late-trial activity appeared quite similar in both the saccade and fixation tasks, this suggests that it is not dependant on the behavioral relevance of the auditory stimulus per se. However, the proportion of cells found to have statistically significant late-trial activity was somewhat lower in the fixation task than the saccade task (39 vs 50\%, respectively, of the cells tested for both tasks). This difference leaves some room for the possibility that sensorimotor or cognitive mechanisms involved in oculomotor localization may recruit more IC neurons for the reward-related signal than those active during passively listening. Additional studies designed to assess how reward- and attention-related signals differ in their influences on sensory processing will be needed to fully answer this important issue (Maunsell, 2004).

The manipulation of reward value revealed an increased activity level on high-reward blocks throughout the trial, not just immediately before the reward delivery. It is possible that there are two signals at play here: one that is steady over the time course of the trial and varies across (blocks of) trials with reward size and another that varies in time in anticipation of the specific moment that the reward will be delivered. Either or both may also be correlated with other cognitive or physiological variables, such as arousal. It will also be of interest to investigate fluctuations in IC responsiveness as the animal's motivational state approaches satiety toward the end of the testing session.

One issue to be resolved relates to the responses occurring at the time of reward delivery. It has been shown that neurons in other brain areas that exhibit a reward expectation signal also respond to reward stimulus (Schultz et al., 1997; Sato and Hikosaka, 2002; Satoh et al., 2003). Although we observed activity during the delivery of the reward, the conditions of our experiments do not permit identification of its cause, which could be acoustic (the sound of the solenoid or the animal's own licking and swallowing sounds) or electrical artifact attributable to the licking movements. Additional experiments that control for the acoustic and artifactual components of the reward delivery will be needed to establish whether there is a response to the delivery of the reward that transcends its specific sensory components.

These findings expand the list of nonauditory influences over primate IC neural activity to include a signal that may be related to reward anticipation. Such a signal may be important for calibrating IC auditory responses according to the cognitive or behavioral context in which sounds are presented. The presence of a signal related to positive reinforcement in the IC is an important piece in the puzzle concerning IC function. The IC has been implicated in the visual calibration of acoustic space in barn owls reared with displacing prisms (Brainard and Knudsen, 1993). How visual stimulation comes to alter the representation of sound location via changes in the connectivity of IC neurons is not completely understood, and a reinforcement signal for correctly localizing a sound source may be vital for this process. That the reward signal is present in broader contexts than just sound 
localization suggests that it may play a role in calibrating or reinforcing whatever other auditory perceptual capacities might be mediated in part by IC activity, such as pitch perception or auditory scene analysis.

Future work will be needed to elucidate precisely what role this signal might play in auditory perception. It will be of particular interest to use a behavioral task in which the animal is actively adjusting its sound localization or other auditory task performance during the course of the session. Such a paradigm might reveal a connection between correct performance and fluctuations in this signal.

\section{References}

Adams JC (1980) Crossed and descending projections to the inferior colliculus. Neurosci Lett 19:1-5.

Andersen RA, Snyder RL, Merzenich MM (1980) The topographic organization of corticocollicular projections from physiologically identified loci in the AI, AII, and anterior auditory cortical fields of the cat. J Comp Neurol 191:479-494.

Bajo VM, Moore DR (2005) Descending projections from the auditory cortex to the inferior colliculus in the gerbil, Meriones unguiculatus. J Comp Neurol 486:101-116.

Brainard MS, Knudsen EI (1993) Experience-dependent plasticity in the inferior colliculus: a site for visual calibration of the neural representation of auditory space in the barn owl. J Neurosci 13:4589-4608.

Brosch M, Selezneva E, Scheich H (2005) Nonauditory events of a behavioral procedure activate auditory cortex of highly trained monkeys. J Neurosci 25:6797-6806.

Coleman JR, Clerici WJ (1987) Sources of projections to subdivisions of the inferior colliculus in the rat. J Comp Neurol 262:215-226.

Cooper MH, Young PA (1976) Cortical projections to the inferior colliculus of the cat. Exp Neurol 51:488-502.

Diamond IT, Jones EG, Powell TP (1969) The projection of the auditory cortex upon the diencephalon and brain stem in the cat. Brain Res 15:305-340.

Doubell TP, Baron J, Skaliora I, King AJ (2000) Topographical projection from the superior colliculus to the nucleus of the brachium of the inferior colliculus in the ferret: convergence of visual and auditory information. Eur J Neurosci 12:4290-4308.

Durif C, Jouffrais C, Rouiller EM (2003) Single-unit responses in the auditory cortex of monkeys performing a conditional acousticomotor task. Exp Brain Res 153:614-627.

Egorova M, Ehret G, Vartanian I, Esser KH (2001) Frequency response areas of neurons in the mouse inferior colliculus. I. Threshold and tuning characteristics. Exp Brain Res 140:145-161.

FitzPatrick KA, Imig TJ (1978) Projections of auditory cortex upon the thalamus and midbrain in the owl monkey. J Comp Neurol 177:573-655.

Groh JM, Trause AS, Underhill AM, Clark KR, Inati S (2001) Eye position influences auditory responses in primate inferior colliculus. Neuron 29:509-518.

Groh JM, Kelly KA, Underhill AM (2003) A monotonic code for sound azimuth in primate inferior colliculus. J Cogn Neurosci 15:1217-1231.

Hage SR, Ehret G (2003) Mapping responses to frequency sweeps and tones in the inferior colliculus of house mice. Eur J Neurosci 18:2301-2312.

Harting JK (1977) Descending pathways from the superior collicullus: an autoradiographic analysis in the rhesus monkey (Macaca mulatta). J Comp Neurol 173:583-612.

Huffman RF, Henson OW (1990) The descending auditory pathway and acousticomotor systems: connections with the inferior colliculus. Brain Res Rev 15:295-323.

Hurley LM, Pollak GD (1999) Serotonin differentially modulates responses to tones and frequency-modulated sweeps in the inferior colliculus. J Neurosci 19:8071-8082.

Hurley LM, Pollak GD (2001) Serotonin effects on frequency tuning of inferior colliculus neurons. J Neurophysiol 85:828-842.

Hurley LM, Pollak GD (2005a) Serotonin shifts first-spike latencies of inferior colliculus neurons. J Neurosci 25:7876-7886.

Hurley LM, Pollak GD (2005b) Serotonin modulates responses to species- specific vocalizations in the inferior colliculus. J Comp Physiol A Neuroethol Sens Neural Behav Physiol 191:535-546.

Ikeda T, Hikosaka O (2003) Reward-dependent gain and bias of visual responses in primate superior colliculus. Neuron 39:693-700.

Itaya SK, Van Hoesen GW (1982) Retinal innervation of the inferior colliculus in rat and monkey. Brain Res 233:45-52.

Judge SJ, Richmond BJ, Chu FC (1980) Implantation of magnetic search coils for measurement of eye position: an improved method. Vision Res 20:535-538.

Komura Y, Tamura R, Uwano T, Nishijo H, Kaga K, Ono T (2001) Retrospective and prospective coding for predicted reward in the sensory thalamus. Nature 412:546-549.

Komura Y, Tamura R, Uwano T, Nishijo H, Ono T (2005) Auditory thalamus integrates visual inputs into behavioral gains. Nat Neurosci 8:1203-1209.

Kuwabara N, Zook JM (2000) Geniculo-collicular descending projections in the gerbil. Brain Res 878:79-87.

Larue DT, Prieto JJ, Winer JA (2005) The central gray and the inferior colliculus: an auditory-limbic interface. Assoc Res Otolaryngol Abstr 288.

Marsh RA, Fuzessery ZM, Grose CD, Wenstrup JJ (2002) Projection to the inferior colliculus from the basal nucleus of the amygdala. J Neurosci 22:10449-10460.

Maunsell JHR (2004) Neuronal representations of cognitive state: reward or attention? Trends Cogn Sci 8:261-265.

Metzger RR, Mullette-Gillman OA, Underhill AM, Cohen YE, Groh JM (2004) Auditory saccades from different eye positions in the monkey: implications for coordinate transformations. J Neurophysiol 92:2622-2627.

Moriizumi T, Leduc-Cross B, Wu JY, Hattori T (1992) Separate neuronal populations of the rat substantia nigra pars lateralis with distinct projection sites and transmitter phenotypes. Neuroscience 46:711-720.

Olazabal UE, Moore JK (1989) Nigrotectal projection to the inferior colliculus: horseradish peroxidase transport and tyrosine hydroxylase immunohistochemical studies in rats, cats, and bats. J Comp Neurol 282:98-118

Paloff AM, Usunoff KG, Hinova-Palova DV, Ivanov DP (1985) Retinal innervation of the inferior colliculus in adult cats: electron microscopic observations. Neurosci Lett 54:339-344.

Porter KK, Metzger RR, Groh JM (2006) The representation of eye position in primate inferior colliculus. J Neurophysiol 95:1826-1842.

Robinson D (1963) A method of measuring eye movement using a scleral search coil in a magnetic field. IEEE Trans Biomed Eng 10:137-145.

Ryan A, Miller J (1977) Effects of behavioral performance on single-unit firing patterns in inferior colliculus of the rhesus monkey. J Neurophysiol 40:943-956.

Ryan A, Miller J (1978) Single unit responses in the inferior colliculus of the awake and performing rhesus monkey. Exp Brain Res 32:389-407.

Sato M, Hikosaka O (2002) Role of primate substantia nigra pars reticulata in reward-oriented saccadic eye movement. J Neurosci 22:2363-2373.

Satoh T, Nakai S, Sato T, Kimura M (2003) Correlated coding of motivation and outcome of decision by dopamine neurons. J Neurosci 23:9913-9923.

Schultz W (2000) Multiple reward signals in the brain. Nat Rev Neurosci 1:199-207.

Schultz W, Dayan P, Montague PR (1997) A neural substrate of prediction and reward. Science 275:1593-1599.

Sutter ML (2000) Shapes and level tolerances of frequency tuning curves in primary auditory cortex: quantitative measures and population codes. J Neurophysiol 84:1012-1025.

Taberner AM, Liberman MC (2005) Response properties of single auditory nerve fibers in the mouse. J Neurophysiol 93:557-569.

Winer JA, Chernock ML, Larue DT, Cheung SW (2002) Descending projections to the inferior colliculus from the posterior thalamus and the auditory cortex in rat, cat, and monkey. Hear Res 168:181-195.

Yamauchi K, Yamadori T (1982) Retinal projection to the inferior colliculus in the rat. Acta Anat (Basel) 114:355-360.

Yan J, Zhang Y, Ehret G (2005) Corticofugal shaping of frequency tuning curves in the central nucleus of the inferior colliculus of mice. J Neurophysiol 93:71-83.

Yasui Y, Nakano K, Kayahara T, Mizuno N (1991) Non-dopaminergic projections from the substantia nigra pars lateralis to the inferior colliculus in the rat. Brain Res 559:139-144.

Zwiers MP, Versnel H, Van Opstal AJ (2004) Involvement of monkey inferior colliculus in spatial hearing. J Neurosci 24:4145-4156. 